\title{
Evaluating amblyopia during the era of COVID-19
}

\author{
Veronica E. Tamez-Tamez ${ }^{1}$ Raul E. Ruiz-Lozano ${ }^{1,2}$ (D) \\ Received: 17 June 2020 /Revised: 17 June 2020 / Accepted: 24 June 2020 / Published online: 2 July 2020 \\ (C) Springer-Verlag GmbH Germany, part of Springer Nature 2020
}

Dear Editor,

Eye diseases seldomly carry a fatal outcome; however, they do represent high morbidity. During the COVID-19 outbreak, the American Academy of Ophthalmology (AAO) recommended ophthalmologists to cease providing non-urgent care. Also, they provided a list of urgent and emergent procedures required to solve potential sightthreatening conditions [1]. Such procedures are mostly related to retinal, glaucomatous, orbital, oncology, and traumatic eye disease [1]. Moreover, other publications addressing recommendations on how to treat such diseases during the outbreak have emerged. Specific recommendations to evaluate patients at risk of developing amblyopia during the pandemic are currently not available.

Amblyopia, with a prevalence of 1 to $5 \%$ of the global population, represents the most common cause of unilateral vision loss in children [2]. It is defined as an interocular difference of $\geq 2$ lines with a visual acuity (VA) of 20/40 or worse in the amblyopic eye [3]. It is related to an abnormal visual experience, mainly caused by strabismus, anisometropia, and visual deprivation, during the period of visual development (approx. up to 6 years) [3]. Because of a good vision of the sound eye, children rarely note diminished VA in the amblyopic eye, thus, they require prompt intervention in order to avoid permanent vision loss [3]. Furthermore, close followup (between 6 and 8 weeks) is required to ensure adequate treatment effect [3]. In this letter we propose evidence-based recommendations on how to adequately evaluate children

This article is part of the topical collection on Perspectives on COVID-19.

Raul E. Ruiz-Lozano

raule.ruiz91@gmail.com

1 Tecnológico de Monterrey, School of Medicine and Health Sciences, Institute of Ophthalmology and Visual Sciences, Monterrey, Mexico

2 Instituto de Oftalmologia y Ciencias Visuales Centro Medico Zambrano Hellion, Av. Batallon de San Patricio No. 112, Col. Real de San Agustin, 66278 San Pedro Garza García, NL, Mexico during the amblyopic period while reducing COVID-19 transmission (Fig. 1).

\section{- Reasons to seek medical care based on parental home screening}

1. In a darkened room, direct a penlight to the child's both eyes from approximately 18 to $30 \mathrm{in}$. A red reflex must be observed, and it must be symmetric, homogeneous, and with equal brightness in both eyes (Brückner test) [4].

- An absent or unequal reflex might be related to conditions causing visual deprivation and alterations in the fixation pattern (strabismus), respectively [4].

- This test must be done during the first 6 weeks of life since, if congenital cataract is present, a best prognosis is ensured if early surgery is performed [5].

2. Failure to fix and follow objects, faces, and light by the third month of age [5].

3. If the child squeezes his/her eyes in bright light, squints or closes or covers one eye when attempting to focus [5].

4. Lan et al. developed an amblyopia screening package (for children 3-6 years) consisting of a set of VA optotypes in order to test visual function and a 6item questionnaire regarding common amblyopic risk factors [2].

5. Eyelid (e.g., ptosis) abnormalities that obstruct the visual axis [4].

\section{- Reasons for referral to a third level of care center} based on telemedicine (TM) approach

1. Visual deprivation

(a) The use of a wide-field digital camera to obtain images of the external eye (cornea and lens) and retina and further revision by an ophthalmologist via TM is recommended [6]. 


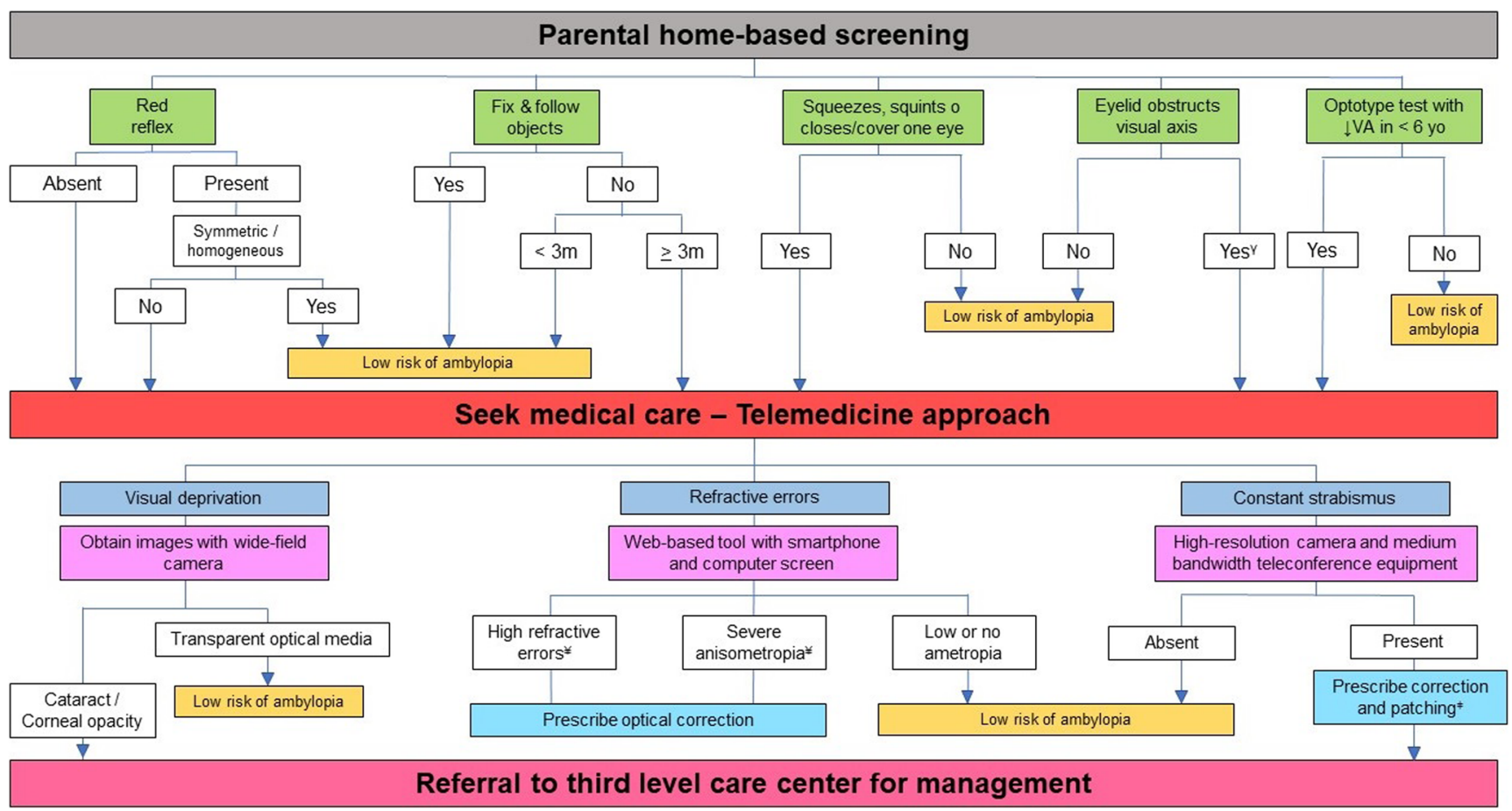

Fig. 1 Block diagram of the proposed approach for amblyopia patients and those at risk of amblyopia during the COVID-19 pandemic. ${ }^{\gamma}$ If an eyelid abnormality is obstructing the visual axis, referral to third level of care center is required. ${ }^{¥}$ Values of severe anisometropia and high refractive errors considered as risk factors of amblyopia are described by Lan et al. [2]. ' If no improvement is observed after 2 months of optical correction and patching, consider referral to third level of care

(c) Patients with amblyopia already followed must receive clear indications to continue using their previously prescribed therapy.

Because of its high prevalence and lifetime morbidity, an adequate and timely management of amblyopia represents an important public health issue. In fact, studies have revealed that amblyopia is one of the most cost-effective procedures in ophthalmology [10]. The hindmost because of its effectiveness in preserving vision and the longevity of this population [10]. Almost 6 months has passed since COVID-19 first appeared, and little do we know when it will end. We must take advantage of the various technological and TM to provide our pediatric population an adequate service during the COVID-19 era.

\section{Compliance with ethical standards}

Conflict of interest The authors declare that they have no conflicts of interest.

\section{References}

1. List of urgent and emergent ophthalmic procedures (2020) American Academy of Ophthalmology. https://www.aao.org/ headline/list-of-urgent-emergent-ophthalmic-procedures. Accessed 16 Jun 2020 
2. Lan W, Zhao F, Li Z, Zeng J, Liu W, Lu J, Zheng D, Lin L, Ge J, Yang Z (2012) Validation and cost-effectiveness of a home-based screening system for amblyopia. Ophthalmology 119(6):12651271. https://doi.org/10.1016/j.ophtha.2011.12.027

3. Chen AM, Cotter SA (2016) The amblyopia treatment studies: implications for clinical practice. Adv Ophthalmol Optom 1(1): 287-305. https://doi.org/10.1016/j.yaoo.2016.03.007

4. Pediatric Ophthalmology/Strabismus Preferred Practice Pattern ${ }^{\circledR}$ (2017) American Academy of Ophthalmology. https://www.aao. org/preferred-practice-pattern/pediatric-eye-evaluations-ppp-2017. Accessed 16 Jun 2020

5. Khokhar S, Pillay G, Agarwal E (2018) Pediatric cataract - importance of early detection and management. Indian J Pediatr 85(3): 209-216. https://doi.org/10.1007/s12098-017-2482-2

6. Simkin SK, Misra SL, Battin M, McGhee CNJ, Dai S (2019) Prospective observational study of universal newborn eye screening in a hospital and community setting in New Zealand. BMJ Pediatr Open 3(1):bmjpo-2018-000376. https://doi.org/10.1136/ bmjpo-2018-000376
7. Wisse RPL, Muijzer MB, Cassano F, Godefrooij DA, Preevo Y, Soeters N (2019) Validation of an independent web-based tool for measuring visual acuity and refractive error (the manifest versus online refractive evaluation trial): prospective open-label noninferiority clinical trial. J Med Internet Res 21(11):e14808. https://doi. org/10.2196/14808

8. Cheung JC, Dick PT, Kraft SP, Yamada J, Macarthur C (2000) Strabismus examination by telemedicine. Ophthalmology 107(11):1999-2005. https://doi.org/10.1016/s0161-6420(00) 00377-8

9. Speeg-Schatz C (2020) Pediatric ophthalmology consultations during COVID-19 pandemic. J Fr Ophthalmol 43(6):547. https://doi. org/10.1016/j.jfo.2020.04.018

10. Busbee BG, Brown GC, Brown MM (2003) Cost-effectiveness of ocular interventions. Curr Opin Ophthalmol 14(3):132-138. https:// doi.org/10.1097/00055735-200306000-00004

Publisher's note Springer Nature remains neutral with regard to jurisdictional claims in published maps and institutional affiliations. 